\title{
Effect of prenatal lignocaine on auditory brain stem evoked response
}

\author{
M E A BOZYNSKI, ${ }^{*}$ R E SCHUMACHER,${ }^{*}$ L S DESCHNER, $†$ AND P KILENY \\ Departments of *Pediatrics and Communicable Diseases, †Anesthesiology, and $\ddagger$ Otolaryngology, \\ University of Michigan Medical Center, Ann Arbor, Michigan, USA
}

SUMmaRY To test the hypothesis that there would be a positive correlation between the interpeak wave (I-V) interval as measured by auditory brain stem evoked response and the ratio of umbilical cord blood arterial to venous lignocaine concentrations in infants born after maternal epidural anaesthesia, 10 normal infants born at full term by elective caesarean section were studied. Umbilical cord arterial and venous plasma samples were assayed for lignocaine, and auditory brain stem evoked responses were elicited at 35 and $70 \mathrm{~dB}$ at $<4$ (test 1 ) and $\geqslant 48$ hours (test 2). Mean wave I-V intervals were prolonged in test 1 when compared with test 2 . Linear regression showed the arterial:venous ratio accounted for $66 \%$ (left ear) and $43 \%$ (right ear) of the variance in test 1 intervals. No association was found in test 2 . In newborn infants, changes in serial auditory brain stem evoked response tests occur after maternal lignocaine epidural anaesthesia and these changes correlate with blood lignocaine concentrations.

Lignocaine, one of the most widely used agents in epidural anaesthesia, has been associated with abnormal neurobehavioural responses in the newborn. ${ }^{1} 2$ In 1974, Scanlon et al reported that lignocaine adversely affected the neonatal behavioural examination (as measured by the Scanlon early neonatal behavioural scale) performed at 2 to 6 hours of life. ${ }^{3}$ Subsequent studies failed to confirm these observations. In general, however, these studies lacked both control for confounding variables and appropriate data analyses. ${ }^{45}$ Several recent studies have confirmed the findings of Scanlon et al, showing decreased response to sound and poor state regulation in infants of mothers receiving lignocaine ${ }^{6}{ }^{9}$ Moreover, experimental studies in animals suggest that lignocaine interferes with neuronal transmission of sound. ${ }^{89}$ Ruth et al found that lignocaine affects the more central portions of the auditory system, especially wave V latency. ${ }^{9}$ This was recently confirmed in newborns by Diaz et al. ${ }^{10}$

We hypothesised that there would be a positive correlation between the interpeak wave (I-V) interval as measured by auditory brain stem evoked response and the ratio of umbilical cord blood arterial:venous lignocaine concentrations in infants born after maternal epidural anaesthesia. The wave (I-V) interpeak interval rather than the absolute wave $\mathrm{V}$ latency was chosen because wave $\mathrm{V}$ latency may be affected by external factors-for example, fluid in the ear canal. On the other hand, the wave I-V interval is a direct, uncontaminated measure of neural conduction along the auditory pathways. The ratio of umbilical cord blood arterial:venous lignocaine concentration was chosen because it better reflects actual tissue concentrations. ${ }^{2}$

\section{Patients and methods}

We studied 10 normal infants born at full term by elective caesarean section after maternal epidural anaesthesia using lignocaine, $64 \mathrm{mmol} / \mathrm{l}$ or $85 \mathrm{mmol} / \mathrm{l}$ (molecular weight lignocaine $=234.3 \mathrm{~g} ; 1 \mathrm{~g}$ lignocaine $=42.6 \mathrm{mmol}$ ). Only low risk mothers with no complications of pregnancy were enrolled. Infants were tested only if their Apgar scores at one and five minutes were $\geqslant 7$, their growth was appropriate for gestational age, they showed no respiratory distress, and they were normal on physical examination in the observation nursery. Informed consent for cord blood lignocaine measurements and auditory brain stem evoked response testing was obtained from one or both parents before the infant's delivery. The study was approved by the Human Investigation Committee of the University of Michigan Medical Center. Presence or absence of replicated responses 
at $35 \mathrm{~dB} \mathrm{nHL}$ (normal hearing level) were determined for each ear. Auditory evoked responses were recorded by surface electrodes attached to the forehead and ipsilateral earlobe or mastoid process with the contralateral ear earthed. Each average consisted of 2000 accepted sweeps. Each averaged response was replicated. The bioelectric activity recorded by the pair of electrodes was amplified differentially $(\times 100000)$ and filtered $(30-3000 \mathrm{~Hz})$. The auditory brain stem evoked responses were recorded and elicited by means of a Nicolet CA-2000 averager.

Auditory brain stem evoked responses were elicited by broad band clicks presented to each ear at 35 and $70 \mathrm{~dB} \mathrm{nHL}$. Each infant was studied at $<4$ (test 1) and $\geqslant 48$ (test 2) hours. In addition, interpeak auditory brain stem evoked response (wave I-V) intervals from the $70 \mathrm{~dB}$ nHL responses were measured by one observer unaware of the infant's cord blood lignocaine concentration and the study hypothesis. The full protocol was repeated for test 2 .

Samples of umbilical cord arterial and venous plasma were assayed for lignocaine using fluorescence immune assay. A segment of umbilical cord was double clamped at delivery, the vessels were identified, and samples were aspirated from each vessel. After centrifugation the plasma was transferred to plastic test tubes and frozen at $-48^{\circ} \mathrm{C}$ until assay could be accomplished. The results were expressed as the ratio of umbilical artery:umbilical venous lignocaine concentrations.

Data were analysed by the Wilcoxon signed rank test; we used a non-parametric statistical test as it could not be assumed that the data were normally distributed (small sample size). The test was two sided. Simple linear regression was also done to study the association between the arterial: venous ratio (predictor variable) and the interpeak (wave I-V) interval measurement (response variable).

\section{Results}

Ten mothers and infants were enrolled in and completed the study. The mean (SD) maternal lignocaine dose was $0.023(0.004) \mathrm{mmol} / \mathrm{kg}$ body weight. Mean (SD) birth weight of the study infants was $3157(421 \mathrm{~g})$ and their mean (SD) gestational age was $39(1 \cdot 1)$ weeks. All were appropriately grown for gestational age. Median Apgar scores at 1 and 5 minutes were 8 (range 7-9) and 9 (all infants), respectively.

Mean interpeak (wave I-V) intervals were prolonged in test 1 compared with test 2 for both ears. The difference was greater for the left ear (left: median 5.4 (range $4 \cdot 6-6 \cdot 0$ ) compared with $5 \cdot 2$
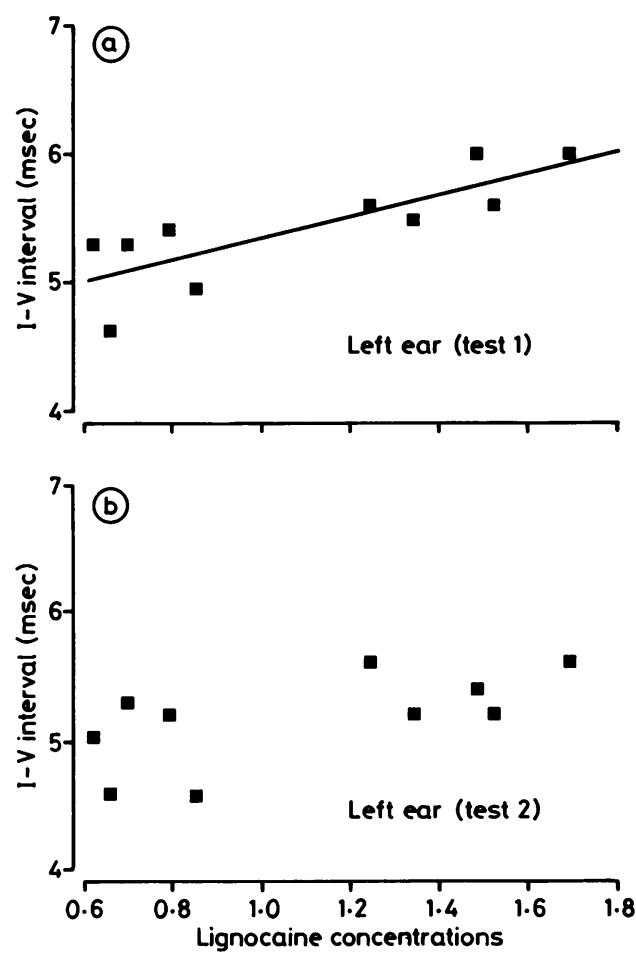

Fig 1(a) Scattergram showing ratio of arterial: venous cord lignocaine concentrations plotted against wave (I-V) interval as measured by auditory brain stem evoked response at $<4$ hours postnatal age (left ear). Linear regression $y=4.519+0.839$ (lignocaine ratio). (b) Ratio of arterial: venous cord lignocaine concentrations plotted against wave (I-V) interval as measured by auditory brain stem evoked response at $\geqslant 48$ hours postnatal age (left ear).

(range 4.5-5.6) msec $(\mathrm{p}<0.01)$ and right: $5 \cdot 4$ (range 4.6-5.8) compared with $5 \cdot 2$ (range 4.6-5.6) $(p=0 \cdot 05))$. Two of three infants with arterial:venous lignocaine ratios more than one standard deviation above the mean had no responses for both ears when tested at $35 \mathrm{~dB}$ (test 1 ). Both infants exhibited replicated auditory brain stem evoked responses to $35 \mathrm{~dB}$ nHL clicks on test 2 .

Mean (SD) plasma lignocaine concentrations were: umbilical artery $3.9(1.2) \mathrm{mmol} / \mathrm{l}$ and umbilical vein 3.8 (1) $\mathrm{mmol} / \mathrm{l}$, arterial:venous ratio being 1.1 $(0.4)$. When simple linear regression analyses were performed, the arterial:venous ratio accounted for $65.8 \%\left(R^{2}\right)$ of the variance in test 1 wave $I-V$ intervals $(r=0 \cdot 81, p=0 \cdot 004)$ as measured in the left ear and $43 \%\left(R^{2}\right)$ of the variance, $(r=0.66, p<0.04)$ in test 1 intervals for the right ear. On retest, the regression analyses no longer showed a significant 

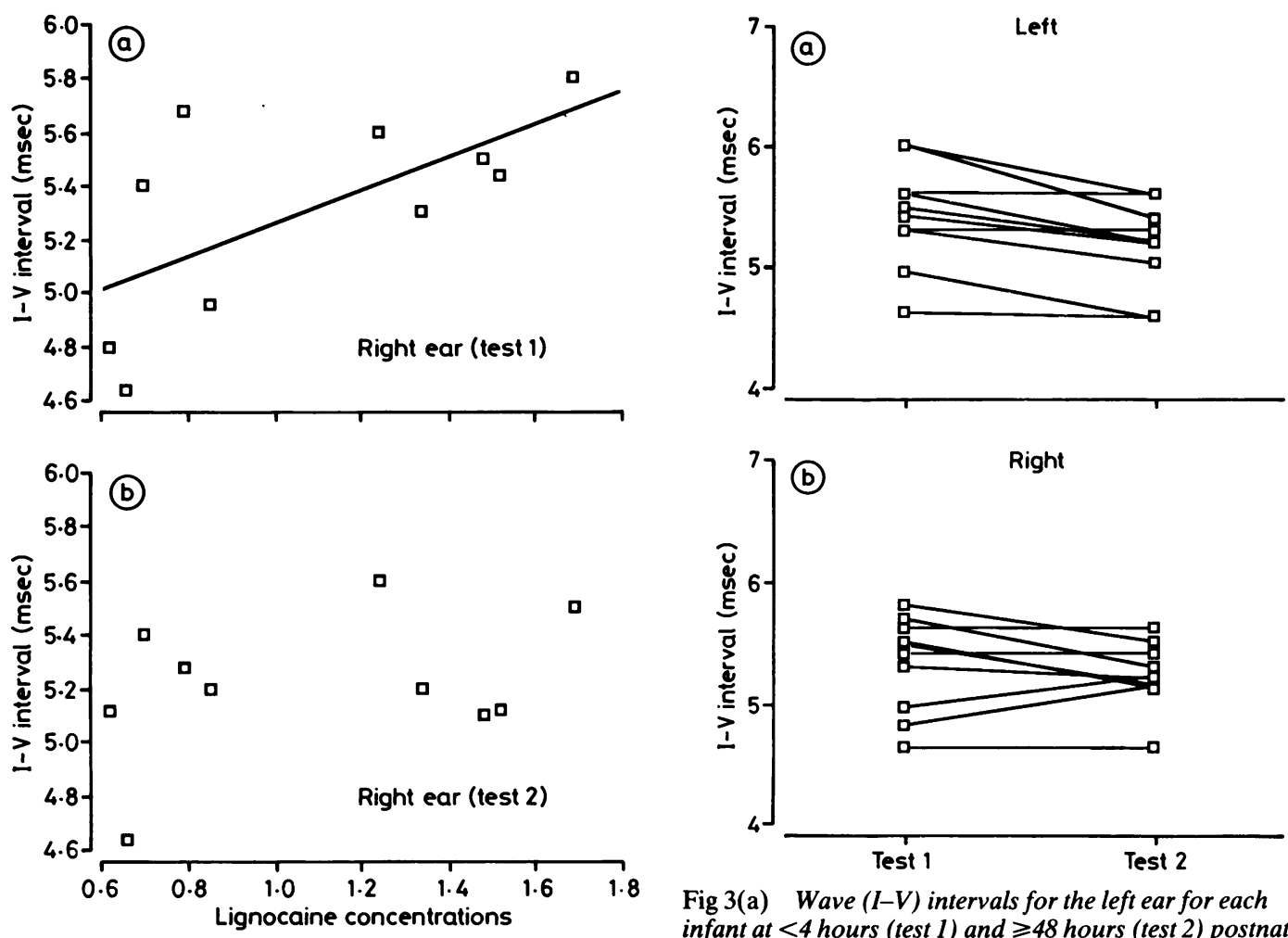

Fig 2(a) Ratio of arterial: venous cord lignocaine concentrations plotted against wave (I-V) interval as measured by auditory brain stem evoked response at $<4$ hours postnatal age (right ear). Linear regression $y=4 \cdot 631+0.626$ (lignocaine concentration). (b) Ratio of arterial: venous cord lignocaine concentrations plotted against wave (I-V) interval as measured by auditory brain stem evoked response at $\geqslant 48$ hours postnatal age (right ear).

relationship between test 2 results and the arterial: venous ratio for either ear.

The results are shown in figs $1-3$.

\section{Discussion}

The results of this study show that there is. an association between cord blood lignocaine concentrations after maternal epidural anaesthesia and prolongation of interpeak (wave I-V) intervals as measured on auditory brain stem evoked response. Furthermore, a dose response relationship is suggested by the test 1 results and arterial:venous lignocaine ratios, as well as by results of the regression analyses. The observed differences between the left and right ears were unexpected.

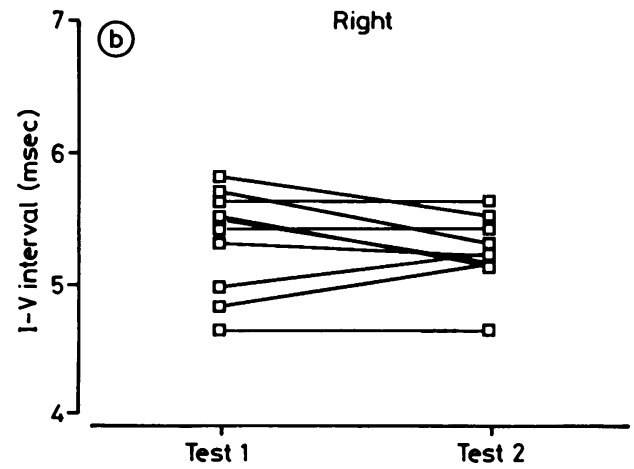

Fig 3(a) Wave (I-V) intervals for the left ear for each infant at $<4$ hours (test 1) and $\geqslant 48$ hours (test 2) postnatal age. (b) Wave (I-V) intervals for the right ear for each infant at $<4$ hours (test 1) and $\geqslant 48$ hours (test 2) postnatal age.

Others have found evidence for a increased vulnerability to hypoxic insult in the left hemisphere compared with the right. ${ }^{11}$ Asymmetry has also been shown for intracranial haemorrhage, left sided periventricular echo densities, ${ }^{12}$ and nonhaemorrhagic ventriculomegaly. ${ }^{14}$ The difference between the left and right ears in this study may be due to asymmetrical vulnerability.

The controversy over the effect of lignocaine anaesthesia on newborn behaviour began with the report of Scanlon et al in $1974 .{ }^{3}$ In addition to decreased hearing responses, they found lower test scores on muscular strength and tone. Ostfeld et al studied the effect of prenatal lignocaine on hearing responses in the infant born at full term at 4 and 24 hours of age. ${ }^{7}$ At both times, they found an abnormal response to sound in $68 \%$ of infants, and hypothesised that lignocaine caused a conduction delay in the eighth nerve or other neuronal pathways.

The effect of lignocaine on neural conduction in 
cats was studied using the auditory brain stem evoked response by Javel et al. ${ }^{8}$ The auditory brain stem evoked response was recorded after systemic infusion of lignocaine at varying rates. Lignocaine's principal effect on the auditory brain stem evoked response was to lengthen all interpeak intervals without reduction of amplitude. The effects were reversible as the drug concentration fell. They hypothesised that lignocaine produced a uniform, dose dependent shift in central nervous impulse conduction times. They further hypothesised that this shift should have its greatest effect on sensory activities requiring higher central processing, such as speech discrimination. Ruth et al recorded the auditory brain stem evoked responses of six healthy male volunteers during intravenous infusion of lignocaine and observed both significant decreases in wave $\mathrm{V}$ amplitude and increases in wave $\mathrm{V}$ latency. ${ }^{9}$

More recently, Diaz et al examined the effect of maternal lignocaine anaesthesia on the auditory brain stem evoked response in 16 infants born at full term. ${ }^{10}$ Eight infants were delivered by caesarean section and exposed to lignocaine, while eight were delivered vaginally without maternal anaesthesia. An auditory brain stem evoked response was obtained on all infants at 3-5 hours, and all infants were tested at 30,60 , and $90 \mathrm{~dB}$. A delay in the wave I-V interpeak interval was seen at $90 \mathrm{~dB}$ in both ears for the neonates exposed to lignocaine. Surprisingly, no effect was seen at $60 \mathrm{~dB}$. As distortion and cross over to the other ear are more likely at $90 \mathrm{~dB}$, the validity of these findings is not clear. In contrast to our findings, no correlation between lignocaine concentrations and prolongation of wave I-V interpeak intervals was seen. This lack of correlation may be explained by their using absolute umbilical artery or vein lignocaine concentrations as opposed to the arterial:venous ratio that more accurately reflects tissue lignocaine concentration. ${ }^{2}$ In our study the mean arterial:venous ratio was greater than 1, indicating that equilibrium between the infants' tissue and blood lignocaine concentrations had been reached. It is possible that the lignocaine arterial: venous ratios were lower in the infants studied by Diaz et al. An additional difference from our study was that Diaz et al reported no follow up auditory brain stem evoked response results to strengthen the causal hypothesis. Nevertheless, both studies suggest an effect of lignocaine.

Our observational study is limited because of the lack of a control group. The ideal control group would include infants delivered in a similar manner - that is, caesarean section without anaesthesia, which is not possible. All low risk elective caesarean sections are carried out under epidural anaesthesia in our hospital, so we were unable to identify a comparison group also delivered by caesarean section. Even if we were to disregard the possible effect of mode of delivery on auditory brain stem evoked response testing, significant plasma lignocaine concentrations have been found after its use for paracervical block and episiotomy. ${ }^{15} 16 \mathrm{We}$ could not, therefore, identify a sufficient number of deliveries for which no anaesthetic agent was used.

Despite the lack of a control group, our data suggest a causal relationship between epidural lignocaine anaesthesia and alteration in the auditory brain stem evoked response in that (a) there was an association, with a significant dose response gradient, between plasma lignocaine arterial:venous values and interpeak intervals; (b) the cause (lignocaine) preceded the effect; (c) altering the cause (lower plasma lignocaine concentrations) altered the effect (decreased interpeak intervals); and (d) the changes in I-V interpeak intervals from the first few hours to the first few days of life cannot be related to neuromaturation, as maturational changes of this magnitude in interpeak intervals usually take four to six months.

Others have, by behavioural testing, shown an association between maternal lignocaine anaesthesia and several newborn behavioural responses. In that lignocaine has been shown to have a effect on all cerebral neural transmission, our data refine and complement those of Diaz et al and support the concept of reported alterations in behavioural responses. As pharmacologically active lignocaine metabolites have been measured in neonatal urine for up to 72 hours, ${ }^{17}$ any impact of lignocaine on behaviour or hearing responses in the neonate should be confined to this time period.

\section{References}

1 Avard DM, Nimord CM. Risk and benefits of obstetric epidural analgesia: a review. Birth 1985;12:215-25.

2 Kuhnert BR, Linn PL, Kuhnert PM. Obstetric medication and neonatal behavior. Current controversies. Clin Perinatol 1985; 12:423-40.

${ }^{3}$ Scanlon JWS, Brown WU, Weiss JB, Alper MH. Neurobehavioral responses of newborn infants after maternal epidural anesthesia. Anesthesiology 1974;40:121-8.

4 Abboud TK, Sarkis F, Blikian A, et al. Lack of adverse neonatal neurobehavioral effects of lidocaine. Anesth Analg 1983;62: 473-5.

5 Kileff ME, James FM, Dewan DM, Floyd HM. Neonatal neurobehavioral responses after epidural anesthesia for cesarian section using lidocaine and bupivicaine. Anesth Analg 1981;63: 413-7.

${ }^{6}$ Kuhnert BR, Harrison MJ, Linn PL, Kuhnert PM. Effects of maternal epidural anesthesia on neonatal behavior. Anesth Analg 1984;63:301-8.

7 Ostfeld B, Friedman S, Menke T, et al. The effect of prenatal lidocaine on hearing responses in term infants. Pediatr Res 1985;19:356A. 
8 Javel E, Mouney DF, McGee J, Walsh EJ. Auditory brainstem responses during systemic infusion of lidocaine. Otolaryngology 1982;108:71-6.

9 Ruth R, Gral TJ, DiFazio CZ, Moscicki JC. Brain-stem auditory-evoked potentials during lidocaine infusion in humans. Archives of Otolaryngology 1985;111:799-802.

10 Diaz M, Graff M, Hiatt IM, Friedman S, Ostfeld B, Hegyi T. Prenatal lidocaine and the auditory evoked responses in term infants. Am J Dis Child 1988;142:160-1.

11 Schumacher RE, Barks JDE, Johnston MD, et al. Right-sided brain lesions in infants following extracorporeal membrane oxygenation. Pediatrics 1988;82:155-61.

12 Donn SM, Bowerman RA. Unilateral germinal matrix hemorrhage in the newborn. J Ultrasound Med 1985;4:251-3.

13 Sobel DB, Go M, Vade A. Nonhemorrhagic ventricular enlargement in newborns. Pediatr Res 1986;20:385A.

14 Guzzetta F, Shackelford GD, Volpe S. Periventricular intraparenchymal echodensities in the premature newborn: critical determination of neurologic outcome. Pediatrics 1986;78: 995-1006.

15 Shnider SM, Way EL. Plasma levels of lidocaine (Xylocaine ${ }^{R}$ ) in mother and newborn following obstetrical conduction anesthesia: clinical applications. Anesthesiology 1968;29:951-8.

${ }^{16}$ Murray AD, Dolby RM, Nation RL, Thomas DB. Effects of epidural anesthesia on newborns and their mothers. Child Dev 1981;52:71-82.

17 Kuhnert BR, Knapp DR, Kuhnert PM, Prochaska BS. Maternal, fetal, and neonatal metabolism of lidocaine. Clin Pharmacol Ther 1979;26:213-20.

Correspondence and requests for reprints to Dr MEA Bozynski, Department of Pediatrics, University of Michigan Medical Center, L 3023 Women's Hospital/0254, 1500 East Medical Center Drive, Ann Arbor, Michigan 48109-0254, USA.

Accepted 11 March 1989 